\title{
THE 2010 MAY FLARING EPISODE OF CYGNUS X-3 IN RADIO, X-RAYS, AND $\gamma$-RAYS
}

\author{
Peter K. G. Williams ${ }^{1}$, John A. Tomsick ${ }^{2}$, Arash Bodaghee ${ }^{2}$, Geoffrey C. Bower ${ }^{1}$, Guy G. Pooley ${ }^{3}$, \\ Katja Pottschmidt ${ }^{4,5}$, Jérôme Rodriguez ${ }^{6}$, Jörn Wilms ${ }^{7}$, Simone Migliari $^{8}$, and Sergei A. Trushirin ${ }^{9}$ \\ ${ }^{1}$ Department of Astronomy, 601 Campbell Hall 3411, University of California, Berkeley, CA 94720-3411, USA; pwilliams@ astro.berkeley.edu \\ ${ }^{2}$ Space Sciences Laboratory, 7 Gauss Way, University of California, Berkeley, CA 94720-7450, USA \\ ${ }^{3}$ The University of Cambridge, Mullard Radio Astronomy Observatory, Cavendish Laboratory, J. J. Thomson Avenue, Cambridge CB3 0HE, UK \\ ${ }^{4}$ CRESST and NASA-Goddard Space Flight Center, Astrophysics Science Division, Astroparticle Physics Laboratory, Greenbelt, MD 20771, USA \\ ${ }^{5}$ CSST, University of Maryland Baltimore County, 1000 Hilltop Circle, Baltimore, MD 21250, USA \\ ${ }^{6}$ AIM-Astrophysique Instrumentation Modélisation (UMR 7158 CEA/CNRS/Université Paris 7 Denis Diderot), CEA Saclay, DSM/IRFU/Service \\ d'Astrophysique, Bât. 709, L'Orme des Merisiers, FR-91 191 Gif-sur-Yvette Cedex, France \\ ${ }^{7}$ Dr. Karl Remeis-Sternwarte and Erlangen Center for Astroparticle Physics, Universität Erlangen-Nürnberg, Sternwartstr. 7, 96049 Bamberg, Germany \\ ${ }^{8}$ Departament d'Astronomia i Meteorologia (DAM) and Institut de Ciènces del Cosmos (ICC), Universitat de Barcelona, Martí i Franquès 1, 08028 Barcelona, Spain \\ ${ }^{9}$ Special Astrophysical Observatory RAS, Karachaevo-Cherkassian res, Nizhnij Arkhyz 36916, Russia \\ Received 2011 March 11; accepted 2011 April 20; published 2011 May 5
}

\begin{abstract}
In 2009, Cygnus X-3 (Cyg X-3) became the first microquasar to be detected in the $\mathrm{GeV} \gamma$-ray regime, via the satellites Fermi and AGILE. The addition of this new band to the observational toolbox holds promise for building a more detailed understanding of the relativistic jets of this and other systems. We present a rich data set of radio, hard and soft X-ray, and $\gamma$-ray observations of Cyg X-3 made during a flaring episode in 2010 May. We detect a $\sim 3$ day softening and recovery of the X-ray emission, followed almost immediately by a $\sim 1$ Jy radio flare at $15 \mathrm{GHz}$, followed by a $4.3 \sigma \gamma$-ray flare $(E>100 \mathrm{MeV}) \sim 1.5$ days later. The radio sampling is sparse, but we use archival data to argue that it is unlikely the $\gamma$-ray flare was followed by any significant unobserved radio flares. In this case, the sequencing of the observed events is difficult to explain in a model in which the $\gamma$-ray emission is due to inverse Compton scattering of the companion star's radiation field. Our observations suggest that other mechanisms may also be responsible for $\gamma$-ray emission from Cyg X-3.
\end{abstract}

Key words: black hole physics - X-rays: binaries - X-rays: individual (Cygnus X-3)

\section{INTRODUCTION}

The X-ray binary (XRB) system Cygnus X-3 (hereafter Cyg X-3), discovered by Giacconi et al. (1967), is notable for its Wolf-Rayet (W-R) companion (van Kerkwijk et al. 1996), short (4.8 hr) orbital period (Bonnet-Bidaud \& Chardin 1988), and dramatic radio variability (Waltman et al. 1994). The relativistic jets that it produces (Geldzahler et al. 1983) classify the source as a "microquasar" system. The distance to Cyg X-3 is $\sim 7$ or $\sim 9 \mathrm{kpc}$, depending on the method used (Predehl et al. 2000; Ling et al. 2009). It is also uncertain whether the compact object is a black hole (e.g., Cherepashchuk \& Moffat 1994) or neutron star (e.g., Stark \& Saia 2003).

Cyg X-3 became more notable in 2009 when it became the first microquasar to be detected in the $\mathrm{GeV} \gamma$-ray regime, via the satellites Fermi (Abdo et al. 2009) and AGILE (Tavani et al. 2009). A $4.8 \mathrm{hr}$ modulation in the Fermi data makes the detection definitive. Emission of $\gamma$-rays has been detected by both observatories during every "high/soft" X-ray state to occur since they began science operations (Abdo et al. 2009; Tavani et al. 2009; Corbel \& Hays 2010; Section 3). The high/soft state is also associated with radio flaring and is closely tied to the launching of relativistic jets (e.g., Fender et al. 2004; Koljonen et al. 2010).

Various physical processes are expected to be involved in the emission and propagation of high-energy photons in Cyg X-3. Inverse Compton (IC) upscattering of the intense $\left(\sim 10^{5} \mathrm{erg} \mathrm{cm}^{-3}\right)$ radiation field of the W-R companion by highenergy $\left(\gamma \gtrsim 10^{3}\right)$ electrons in the relativistic jet is the most efficient source of $\mathrm{GeV}$ emission (Abdo et al. 2009; Dubus et al. 2010). If the density of relativistic protons in the jet is sufficiently high, inelastic collisions between these and protons in the stellar wind will produce neutral pions that decay into $\gamma$-rays (among other products) as well (Romero et al. 2003). In this case, there will be further $\gamma$-ray emission due to secondary leptons created during the hadronic interactions (Orellana et al. 2007). The typical hadronic content of microquasar jets is unknown: large numbers of hadrons impose significant energetic constraints, but there is direct evidence of their presence in at least one system, SS 433 (Kotani et al. 1994, 1996; Migliari et al. 2002).

A $\gamma$-ray flare due to IC upscattering by jet electrons will be followed by a radio flare a few days later as those electrons cool, rarify, and become transparent to their radio synchrotron emission. This phenomenon has been observed repeatedly but not invariably (Abdo et al. 2009; Tavani et al. 2009). If the $\gamma$-ray emission is primarily hadronic, the result will be substantially the same, as secondary processes will create a similar population of energetic leptons. Abdo et al. (2009) determine an approximate $5 \pm 7$ days lag between radio and $\gamma$-ray emission. Inspection of their Figure 2 and the data presented by Tavani et al. (2009) suggests that this uncertainty is primarily systematic rather than statistical; i.e., the radio $/ \gamma$-ray lag varies significantly from event to event. Blazar observations often show lag variability, both between sources and in the same source over time, with theoretical support for sign changes in the lag between $\gamma$-ray and other bands (Aller et al. 2010).

In 2010 May, multiple monitoring programs detected that Cyg X-3 was becoming more active in $\gamma$-rays and was entering the high/soft state (Bulgarelli et al. 2010; Corbel \& Fermi Large Area Telescope Collaboration 2010; Kawai et al. 2010; Corbel $\&$ Hays 2010). We describe multiband observations of Cyg X-3 made during this episode (Section 2) and present our results (Section 3). Our data show a $\gamma$-ray flare apparently lagging a radio flare, a sequencing opposite of that expected in the typical 
interpretation. We discuss and interpret this result (Section 4) and, finally, present our conclusions (Section 5).

\section{OBSERVATIONS AND DATA ANALYSIS}

We observed Cyg X-3 during its 2010 May flaring episode with a variety of instruments. Below, we describe the observations and their analysis. Many dates and times we mention are near MJD 55,340. For brevity, we express these in an offset MJD, defining OJD $=$ MJD $-55,300$. Thus, OJD 0 is JD 2,455,300.5 or 2010 April 14.0 UT, and the $\gamma$-ray flare was detected on OJD $43=2010$ May 27 UT.

\subsection{Radio}

The Arcminute Microkelvin Imager (AMI) arrays (Cambridge, UK) are two aperture synthesis telescopes mainly used to study the cosmic microwave background (Zwart et al. 2008). The observations described herein were made with the Large Array (AMI-LA), the reconfigured and reequipped Ryle Telescope, consisting of eight $13 \mathrm{~m}$ antennas with a maximum baseline of about $120 \mathrm{~m}$, observing in the band 12-18 GHz. The angular resolution is typically $25^{\prime \prime}$. Monitoring of smalldiameter sources is undertaken as described in Pooley \& Fender (1997); observations are interleaved with those of a phasereference calibrator, and after calibration the data for individual baselines are vector-averaged. The in-phase component then provides an unbiased estimate of the target source's flux density. The amplitude scale was calibrated by (at least) daily observations of 3C 48 and 3C 286, both of which are believed to be very nearly constant on long timescales.

Should there be emission on a scale resolved by some of the baselines, the flux density estimate would be incorrect. There is an extended region of low-brightness emission around Cyg X-3 (Sánchez-Sutil et al. 2008), which is detected on the shortest baselines at extreme hour angles with the AMI-LA, and is sufficiently bright to need separate treatment when the central source in Cyg X-3 is "quenched" (flux density $\lesssim 10 \mathrm{mJy}$ at $15 \mathrm{GHz}$ ). No such correction was required in the observations described here.

Observations of Cyg X-3 were also made with the RATAN-600 telescope (Nizhnij Arkhyz, Russia) as part of an ongoing microquasar monitoring campaign (Trushkin 2000; Trushkin et al. 2006). The observations and data analysis were performed as described in Trushkin et al. (2006). In this work, we show results at $11.2 \mathrm{GHz}$; near-simultaneous observations were made at 4.8 and $7.7 \mathrm{GHz}$ as well.

Cyg X-3 was observed by the Allen Telescope Array (ATA; Northern California, USA; Welch et al. 2009) six times in the period OJD 26-43 as part of a larger transient search. Continuum images at $3.09 \mathrm{GHz}$ were made after subtraction of model of the static sky. Source fluxes were determined in $\sim 10$ minute segments by fitting point source models in the image domain.

\subsection{X-ray}

Our study includes hard X-ray observations made with the International Gamma-Ray Astrophysics Laboratory (INTE$G R A L$ ) satellite (Winkler et al. 2003). We used the INTEGRAL Soft Gamma-Ray Imager (ISGRI; Lebrun et al. 2003) instrument and included two observations that were part of a program to observe Cyg X-1 (PI: J. Wilms). Although the primary target of the observations was Cyg X-1, the ISGRI field of view (FOV) is large enough to include the entire Cygnus region, and our target was in the FOV throughout the observations. The first observation occurred during revolution 929, starting at OJD 40.625 and ending at OJD 41.940. The second observation occurred during revolution 938, starting at OJD 67.469 and ending at OJD 68.805. A preliminary report of the first observation was given in Tomsick et al. (2010).

We reduced the ISGRI data using the Off-line Scientific Analysis (OSA v9.0) software package. The program ibis_science_analysis is the primary tool for extracting the standard data products. We produced and inspected the ISGRI image in the $20-40 \mathrm{keV}$ band, and then made energy spectra and light curves for all of the bright sources in the field. After correcting for instrumental dead time, the total exposure times for Cyg X-3 from the first and second observations are $62,420 \mathrm{~s}$ and $66,540 \mathrm{~s}$, respectively. We produced $20-40 \mathrm{keV}$ light curves with a time resolution of $1 \mathrm{ks}$.

We also obtained one-day average quick-look measurements from the All-Sky Monitor on the Rossi X-Ray Timing Explorer (RXTE/ASM; Levine et al. 1996) and transient monitor results from the Burst Alert Telescope on the Swift $\gamma$-ray burst mission (Swift/BAT; Barthelmy et al. 2005). These results are provided publicly by the RXTE/ASM and Swift/BAT teams, respectively.

$$
\text { 2.3. } \gamma-\text { Ray }
$$

Fermi Science Tools 9.17 and HEASoft 6.9 were used to reduce and analyze all Fermi-LAT (Large Area Telescope; Atwood et al. 2009) observations within $10^{\circ}$ of Cyg X-3 that took place in the range OJD -59-150 (2010 February 14-2010 September 11 UT), providing a baseline of $\sim 100$ days before and after the flare of OJD 43-44. Cyg X-3 is within the LAT FOV for $\sim 15 \mathrm{ks} \mathrm{day}^{-1}$. Given this and the typical flux of Cyg $\mathrm{X}-3$ in the LAT band, we were compelled to use one-day time bins in the following analysis.

Thirty arcminutes away from Cyg X-3 is a comparatively bright pulsar, PSR J2032+4127. Using gtephem and gtpphase, we added the phase from the most recent ephemeris available (Abdo et al. 2009) to the events file. Then, we extracted events corresponding to the off-pulse phases $(0-0.12,0.2-0.6$, and 0.72-1; Camilo et al. 2009). This removed $20 \%$ of the live time, so the exposure time of the off-pulse events file was corrected accordingly.

With gtselect and gtmktime, good time intervals from the off-pulse events file were selected in the $0.1-10 \mathrm{GeV}$ energy range. At high energies ( $\gtrsim 10 \mathrm{GeV}), \mathrm{Cyg} X-3$ is expected to have negligible emission. The events class was set to "3" which selects only high-quality diffuse-background photons. To minimize background albedo photons from the Earth's limb, zenith and rocking angles were restricted to less than $105^{\circ}$ and $52^{\circ}$, respectively.

Exposure maps were generated by gtexpcube and gtexpmap while gtbin created photon count maps in the region of interest. Emission from Cyg X-3 was not apparent on the count maps, which is unsurprising given that Cyg X-3 is a faint source whose peak emission is at the low end of the LAT band where the pointspread function is $5^{\circ}-10^{\circ}$. Complicating matters is the high level of diffuse background emission.

Following the procedure described in Abdo et al. (2009), unbinned likelihood analysis was performed for the daily bins with gtlike considering photons inside a $7^{\circ}$ radius of Cyg X-3 from all 1FGL (Abdo et al. 2010) sources up to $5^{\circ}$ away, and all bright (detection significance $>7 \sigma$ and flux $[>100 \mathrm{MeV}]$ $>5 \times 10^{-8} \mathrm{ph} \mathrm{cm}^{-2} \mathrm{~s}^{-1}$ ) 1 FGL sources up to $20^{\circ}$ away. The instrument response function was "Pass 6 v3" (P6_V3_DIFFUSE), and the convergence relied on the NEWMINUIT method. 


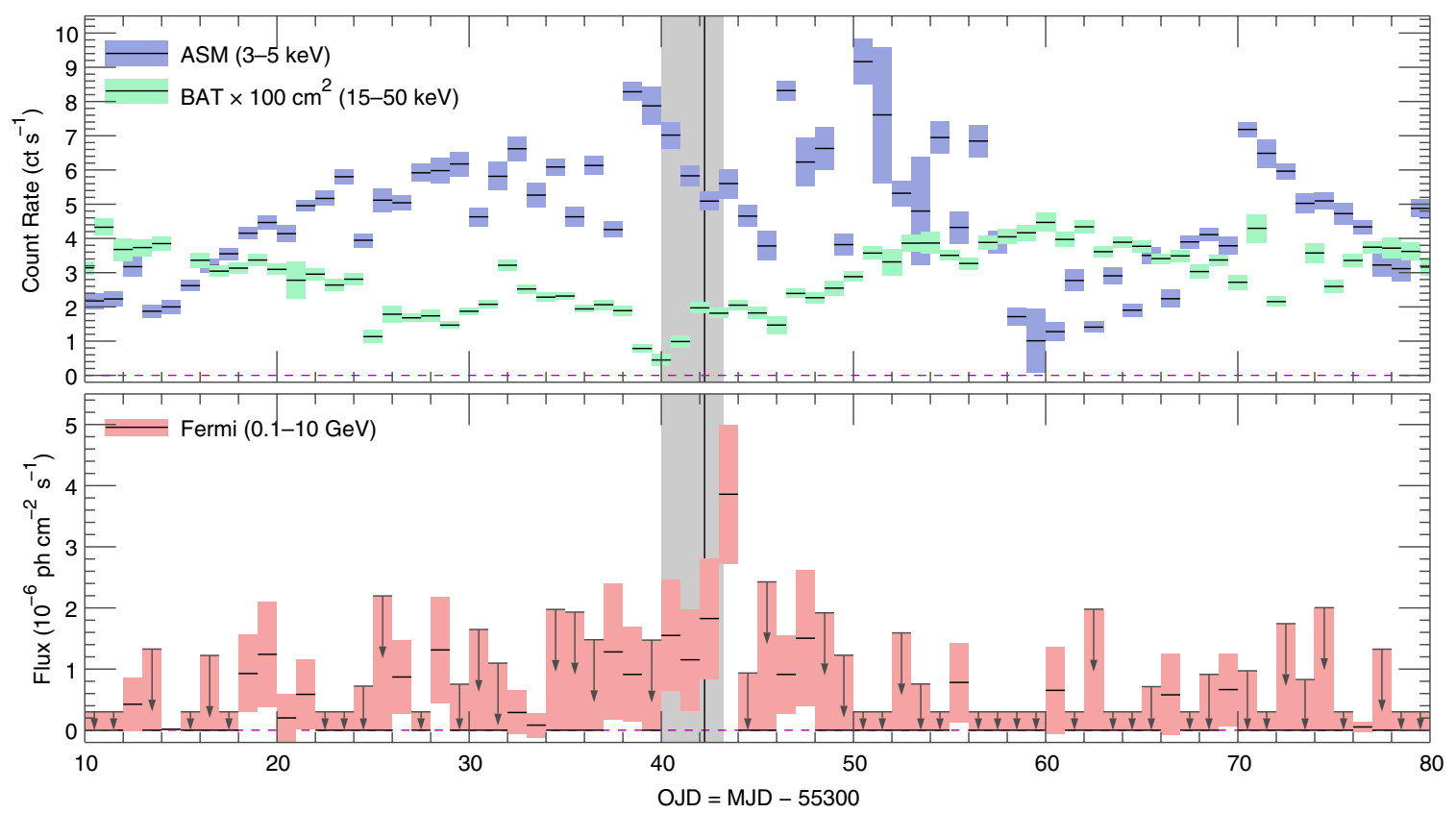

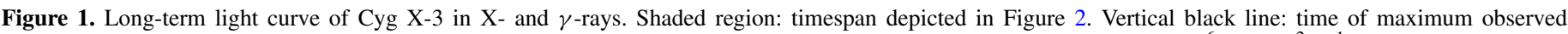

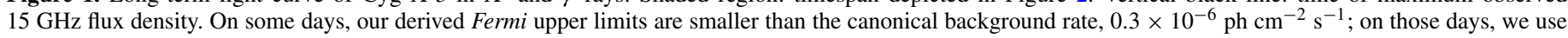
the background rate as the upper limit.

Spectral models and input parameters from the 1FGL catalog were used except for pulsars 1FGL J2021.0+3651 and 1FGL J2021.5+4026 which had exponential cutoffs in addition to their power laws. Besides Cyg X-3, whose photon index and normalization were free, all sources had spectral parameters fixed to the 1FGL values. We included models for the galactic (gll_iem_v02) and extragalactic (isotropic_iem_v02) diffuse emission. The normalizations were left free to vary, as was the photon index of the galactic component.

\section{RESULTS}

Long-term light curves of Cyg X-3 in soft and hard X-ray bands are presented in the top panel of Figure 1. The system was in a soft X-ray state during OJD $~ 20-55$, with an episode of particular softness occurring during OJD 39-41.

The Fermi source flux integrated over $0.1-10 \mathrm{GeV}$ is presented in the lower panel of Figure 1. In bins where the test statistic (TS $\sim-2 \ln L$, where $L$ is the ratio of the likelihood of models without and with the source) is less than one, we compute and plot a $1 \sigma$ upper limit. A peak is seen starting on OJD 43. The TS value in this bin is 18.4 , which translates approximately to $4.3 \sigma$, and the flux is $(4 \pm 1) \times 10^{-6} \mathrm{ph} \mathrm{cm}^{-2} \mathrm{~s}^{-1}$. We are unable to achieve a more precise timing of the flare. Shifting the binning by half a day results in lower-significance and lower-flux detections in the range OJD 42.5-44.5. Analysis using higher-cadence binning does not yield significant detections of the peak.

Radio measurements from just before the $\gamma$-ray flare are presented in the top panel of Figure 2. A $\sim 1 \mathrm{Jy}$ radio flare occurred on OJD 42.25. There is evidence of an earlier flare on OJD 41.11, with a maximum observed flux density of $366 \mathrm{mJy}$, but only the rising portion of the flare is observed, so its properties are poorly constrained. As is shown in Figure 3, the $15 \mathrm{GHz}$ flux density of Cyg X-3 is depressed in the seven days following the $\gamma$-ray flare, with a mean flux density of $48 \mathrm{mJy}$ and an 80th-percentile observed flux density of $66 \mathrm{mJy}$. (That is, $80 \%$ of the measurements during this period are $<66 \mathrm{mJy}$.) Starting on OJD 51, the typical radio flux density increases by a factor of $\sim 2$ to $\sim 100 \mathrm{mJy}$.

The bottom panel of Figure 2 shows high-cadence hard Xray measurements from before the $\gamma$-ray flare, made as Cyg X-3 exited the softest portion of its soft state. They show the recovery of the hard X-ray flux, subject to orbital modulation. The modulation makes it difficult to determine the significance of the drop in flux seen in the last three measurements; it may be evidence of a significant reduction in hard X-ray emission precursing the radio flare. Combining the Swift/ BAT and INTEGRAL measurements, the hard X-ray flux has recovered by OJD $\sim 42.0$, about $5 \mathrm{hr}$ before the observed radio flare. The $\gamma$-ray flare then occurred $\sim 1-2$ days later.

\section{DISCUSSION}

If our observations are taken at face value-that is, one assumes no significant radio activity during coverage gaps and that the flaring stems from one ejection event - the sequencing of the radio and $\gamma$-ray emission is inconsistent with the companion IC model discussed in Section 1. Emission in $\gamma$-rays following a radio flare could be interpreted as a re-energization of the relativistic jet electrons by a shock (Dubus et al. 2010). With a typical jet speed of $\sim 0.5 c$ (Tudose et al. 2007) and a delay of $\sim 1$ day, the re-energization would occur $\sim 100$ AU from the system. This is much larger than the orbital separation but three orders of magnitude smaller than the distance at which the jet is expected to interact significantly with the interstellar medium $(\sim 1 \mathrm{pc})$. Such re-energization could be due to a collision between the jet and a dense clump of the W-R wind (Araudo et al. 2009). In this scenario, the absence of $\gamma$-ray emission at the time of ejection can be explained by absorption within the system (Cerutti et al. 2011), although this model must be reconciled with the observations of Tavani et al. (2009) and Abdo et al. (2009). The re-energization would lead to additional radio emission as the $\gamma$-ray-emitting electrons cool, although the 


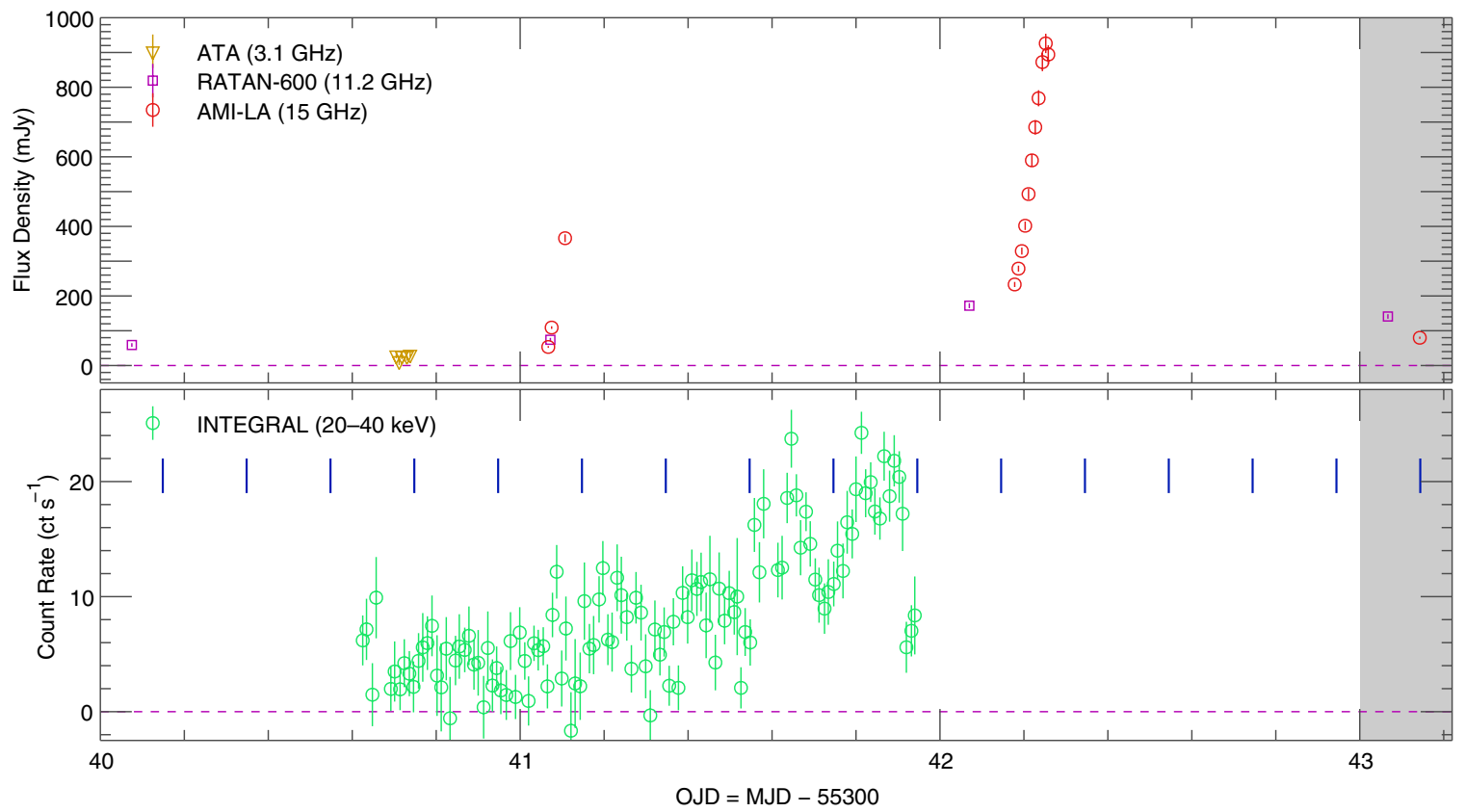

Figure 2. High-cadence light curve of Cyg X-3 in radio and hard X-rays in the time immediately before the $\gamma$-ray peak (= OJD 43-44). The X-rays are subject to orbital modulation with a $4.8 \mathrm{hr}$ periodicity. Vertical blue markers: times of X-ray minima according to parabolic ephemeris of Singh et al. (2002). Shaded region: partial timespan of Fermi peak.
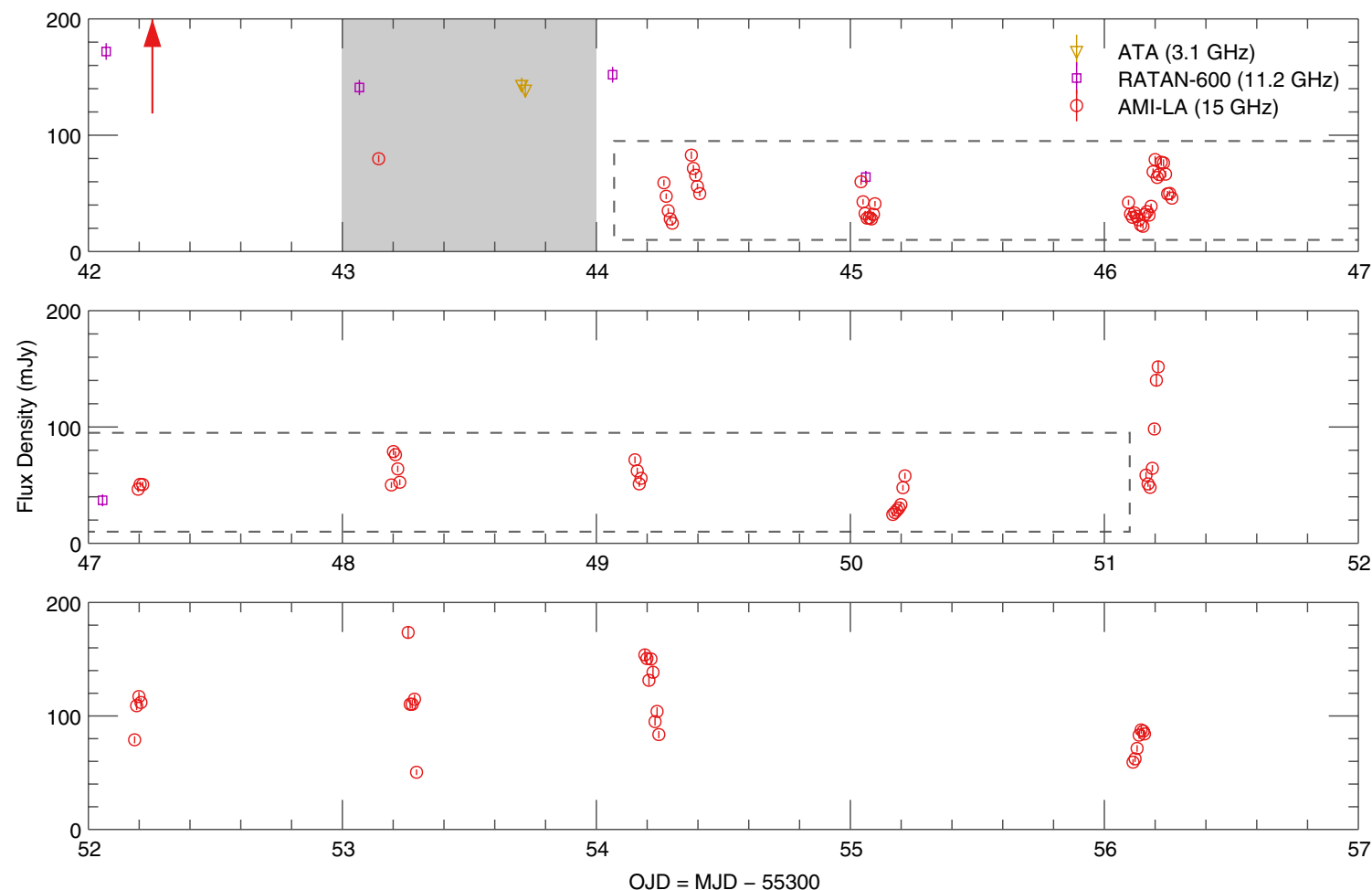

Figure 3. Radio light curve of Cyg X-3 during and after the $\gamma$-ray flare. The three panels proceed in chronological order from top to bottom. Red arrow: time of maximum observed $15 \mathrm{GHz}$ flux density $(=926 \mathrm{mJy})$. Shaded region: timespan of the Fermi peak $\left(=(4 \pm 1) \times 10^{-6} \mathrm{ph} \mathrm{cm}^{-2} \mathrm{~s}^{-1}\right)$. Dashed box: period of quiescent radio emission.

ambient magnetic field, and hence synchrotron luminosity, will be much weaker than that found close to the system.

If the $\gamma$-ray flare is interpreted as the result of a discrete ejection event, the lack of a notable subsequent radio flare could be explained by the ejected material being largely hadronic. While hadronic $\gamma$-ray emission is not as efficient as IC upscattering, it does yield a higher ratio of $\gamma$-ray to radio luminosity (Romero
\& Vila 2008). The secondary leptons due to hadronic interactions would, however, radiate, and detailed studies typically find that their bolometric luminosity is comparable to that of the primaries (e.g., Vila \& Romero 2010).

Should our data be taken at face value? Application of a simple synchrotron-cloud model (van der Laan 1966) supports the intuition that radio flares with sizes comparable to that of 
the largest observed could have occurred without detection, with the modeled lifetimes being $\sim 0.2$ days. While this model has had ambiguous success when applied to Cyg X-3 (Fender et al. 1997), its simplicity is advantageous for our sparse data, and it has been successfully applied to observations of other systems (e.g., Fender et al. 1997; Wilms et al. 2007; Prat et al. 2010). To obtain a better understanding of the radio behavior of Cyg X-3, we compared the 2010 May data to 15 years of archived AMI-LA/Ryle Telescope observations. The $\sim 1 \mathrm{Jy}$ radio flare is unusual: no flux densities $>400 \mathrm{mJy}$ are detected in a 500 day span around the observations we describe.

In light of the predictions of the companion IC model, we consider particularly the radio observations after the observed $\gamma$-ray flare. The duty cycle of the observations is only $6.8 \%$ in the seven-day post-flare quiescent interval. We identified 51 epochs similar to this in the archives, each lasting at least seven days and having an 80th-percentile observed flux less than $70 \mathrm{mJy}$ (cf. Section 3). These archival measurements have a duty cycle comparable to that of the 2010 May data (4.9\%), but a much larger time on-source (55.5 days). Because this time is large compared to the total duration of the 2010 May post$\gamma$-flare quiescence, a search for flares in the archival data set can constrain the likelihood of there having been an unobserved flare in the 2010 May data set, assuming no long-term evolution in the behavior of the quiescent state and stochastic flaring.

The epochs were identified by exhaustively searching for seven-day segments meeting the aforementioned criteria, then lengthening these segments as much as possible without violating the 80th-percentile constraint. Segments separated by less than three days were merged, in a few cases shortening the segment somewhat to preserve the statistical constraint. This method of construction does not bias against epochs containing rapid flares, no matter their size. Qualitatively, the light curve of the typical epoch starts high, drops to very low flux densities, and then becomes high again, possibly with rapid flares in the middle.

The largest flux density seen in the selected archival data was $524 \mathrm{mJy}$, in the context of a single rapidly evolving (rise time $\sim 0.1$ days) flare that would not have necessarily been detected in the 2010 May observing. (Here we exclude slow, large, epoch-terminating flares that would have been easily seen in the 2010 May data.) Approximating the rate of such flares as one per 55.5 days of observing, we derive an $11 \%$ chance that such a flare occurred in the seven days after the $\gamma$-ray peak without being observed. Ignoring the epoch beginnings and endings, which contain high flux values by construction, we find that the flux density of Cyg X-3 is larger than $250 \mathrm{mJy}$ only $0.3 \%$ of the time during its quiescent state.

\section{SUMMARY AND CONCLUSIONS}

We have presented observations of a 2010 May Cyg X-3 flaring episode in the radio, soft and hard X-ray, and $\gamma$-ray bands. Our data show a sequence of three notable events: a particular softening and partial recovery of the X-ray emission, a rapid $\sim 1$ Jy radio flare, and a $\gamma$-ray flare. Interpretation of the data is made more challenging by the sparse sampling of the radio data and the low cadence of the high-energy observations as compared with the rapidity with which the radio emission can evolve. Nonetheless, the observations we do have and comparisons to historical data challenge the interpretation that the $\gamma$-ray emission is due to IC upscattering of the companion radiation field by high-energy electrons leaving the system in a relativistic jet, because there is no evidence for the subsequent radio emission that one would expect to see from these electrons.

While the companion IC model of $\gamma$-ray emission from microquasars is clear and compelling, the lack of consistent radio $/ \gamma$-ray timing lags call into question its completeness. There could be more than one mechanism responsible for the $\gamma$-ray emission of Cyg X-3, becoming more or less relevant in different circumstances, or the reprocessing of the $\gamma$-ray emission by effects such as pair production could be more significant than commonly assumed. Detections of or limits to very high energy $\gamma$-rays $(\gtrsim 100 \mathrm{GeV}$; e.g., Aleksić et al. 2010) or neutrinos from Cyg X-3 would aid in the understanding of the processes relevant to the emission in the Fermi band.

The power of multiband monitoring of Cyg X-3 promises to increase significantly with the addition of $\gamma$-ray data to the set of available observations. As Cyg X-3 inevitably produces more flares, frequent observations at all wavelengths are important to build a detailed understanding of the launching and propagation of its relativistic jets. Radio observations of $\lesssim 2 \mathrm{hr}$ cadence with near-continuous coverage would be ideal for establishing an unambiguous relationship between radio and $\gamma$-ray flaring. Based on the phenomenology we observe, intensive radio observations should be triggered at the ends of very soft X-ray states without waiting for the detection of $\gamma$-ray flares.

The authors thank S. Corbel for useful discussions. P.K.G.W. was supported by a Space Sciences Lab Summer Fellowship. P.K.G.W., J.A.T., and A.B. acknowledge partial support from Fermi and INTEGRAL Guest Investigator grants NNX08AW58G, NNX08AX91G, and NNX10AG50G. S.M. acknowledges financial support from MEC and European Social Funds through a Ramón y Cajal and the Spanish MICINN through grant AYA2010-21782-C03-01. Research with the ATA is supported by the Paul G. Allen Family Foundation, the National Science Foundation, the US Naval Observatory, and other public and private donors. AMI is operated by the University of Cambridge and supported by STFC. This research has made use of NASA's Astrophysics Data System.

Facilities: AMI, ATA, Fermi, INTEGRAL, RATAN, RXTE, Swift

\section{REFERENCES}

Abdo, A. A., et al. 2009, Science, 326, 840

Abdo, A. A., et al. 2010, ApJS, 188, 405

Aleksić, J., et al. 2010, ApJ, 721, 843

Aller, M. A., Hughes, P. A., \& Aller, H. D. 2010, in Fermi Meets Jansky-AGN in Radio and Gamma-rays, ed. T. Savolainen et al. (Bonn: Germany), 65 Araudo, A. T., Bosch-Ramon, V., \& Romero, G. E. 2009, A\&A, 503, 673 Atwood, W. B., et al. 2009, ApJ, 697, 1071

Barthelmy, S. D., et al. 2005, Space Sci. Rev., 120, 143

Bonnet-Bidaud, J. M., \& Chardin, G. 1988, Phys. Rep., 170, 325

Bulgarelli, A., et al. 2010, ATel, 2609, 1

Camilo, F., et al. 2009, ApJ, 705, 1

Cerutti, B., Dubus, G., Malzac, J., Szostek, A., Belmont, R., Zdziarski, A., \& Henri, G. 2011, A\&A, 529, A120

Cherepashchuk, A. M., \& Moffat, A. F. J. 1994, ApJ, 424, L53

Corbel, S. Fermi Large Area Telescope Collaboration.? 2010, ATel, 2611, 1

Corbel, S., \& Hays, E. 2010, ATel, 2646, 1

Dubus, G., Cerutti, B., \& Henri, G. 2010, MNRAS, 404, L55

Fender, R. P., Belloni, T. M., \& Gallo, E. 2004, MNRAS, 355, 1105

Fender, R. P., Burnell, S. J. B., Waltman, E. B., Pooley, G. G., Ghigo, F. D., \& Foster, R. S. 1997, MNRAS, 288, 849

Fender, R. P., Pooley, G. G., Brocksopp, C., \& Newell, S. J. 1997, MNRAS, 290, L65

Geldzahler, B. J., et al. 1983, ApJ, 273, L65

Giacconi, R., Gorenstein, P., Gursky, H., \& Waters, J. R. 1967, ApJ, 148, L119 Kawai, N., et al. 2010, ATel, 2635, 1 
Koljonen, K. I. I., Hannikainen, D. C., McCollough, M. L., Pooley, G. G., \& Trushkin, S. A. 2010, MNRAS, 406, 307

Kotani, T., Kawai, N., Matsuoka, M., \& Brinkmann, W. 1996, PASJ, 48, 619

Kotani, T., et al. 1994, PASJ, 46, L147

Lebrun, F., et al. 2003, A\&A, 411, L141

Levine, A. M., Bradt, H., Cui, W., Jernigan, J. G., Morgan, E. H., Remillard, R., Shirey, R. E., \& Smith, D. A. 1996, ApJ, 469, L33

Ling, Z., Zhang, S. N., \& Tang, S. 2009, ApJ, 695, 1111

Migliari, S., Fender, R., \& Méndez, M. 2002, Science, 297, 1673

Orellana, M., Bordas, P., Bosch-Ramon, V., Romero, G. E., \& Paredes, J. M. 2007, A\&A, 476, 9

Pooley, G. G., \& Fender, R. P. 1997, MNRAS, 292, 925

Prat, L., Rodriguez, J., \& Pooley, G. G. 2010, ApJ, 717, 1222

Predehl, P., Burwitz, V., Paerels, F., \& Trümper, J. 2000, A\&A, 357, L25

Romero, G. E., Torres, D. F., Kaufman Bernadó, M. M., \& Mirabel, I. F. 2003, A\&A, 410, L1

Romero, G. E., \& Vila, G. S. 2008, A\&A, 485, 623

Sánchez-Sutil, J. R., Martí, J., Combi, J. A., Luque-Escamilla, P., Muñoz Arjonilla, A. J., Paredes, J. M., \& Pooley, G. 2008, A\&A, 479, 523
Singh, N. S., Naik, S., Paul, B., Agrawal, P. C., Rao, A. R., \& Singh, K. Y 2002, A\&A, 392, 161

Stark, M. J., \& Saia, M. 2003, ApJ, 587, L101

Tavani, M., et al. 2009, Nature, 462, 620

Tomsick, J. A., et al. 2010, ATel, 2644, 1

Trushkin, S. A. 2000, Astron. Astrophys. Trans., 19, 525

Trushkin, S. A., Bursov, N. N., Nizhelskij, N. A., Majorova, E. K., \& Voitsik, P. A. 2006, in VI Microquasar Workshop: Microquasars and Beyond, ed. T. Belloni (Como: Proc. of Sci.), PoS(MQW6)015

Tudose, V., et al. 2007, MNRAS, 375, L11

van der Laan, H. 1966, Nature, 211, 1131

van Kerkwijk, M. H., Geballe, T. R., King, D. L., van der Klis, M., \& van Paradijs, J. 1996, A\&A, 314, 521

Vila, G. S., \& Romero, G. E. 2010, MNRAS, 403, 1457

Waltman, E. B., Fiedler, R. L., Johnston, K. L., \& Ghigo, F. D. 1994, AJ, 108, 179

Welch, J., et al. 2009, Proc. IEEE, 97, 1438

Wilms, J., Pottschmidt, K., Pooley, G. G., Markoff, S., Nowak, M. A., Kreykenbohm, I., \& Rothschild, R. E. 2007, ApJ, 663, L97

Winkler, C., et al. 2003, A\&A, 411, L1

Zwart, J. T. L., et al. 2008, MNRAS, 391, 1545 